\title{
Efecto del tratamiento de escaldado sobre la actividad enzimática de la polifenoloxidasa en dos variedades de batata (Ipomoea batatas Lam.)
}

\section{Blanching treatment effect on the enzymatic activity of polyphenoloxidase in two varieties of sweet potato (Ipomoea batatas Lam.)}

GUILLERMO ARRÁZOLA-PATERNINA ${ }^{1,3}$

ARMANDO ALVIS-BERMÚDEZ1

CARLOS GARCÍA-MOGOLLÓN²

Batatas coloradas y blancas producidas en el valle del río Sinú.

Foto: G. Arrázola-Paternina

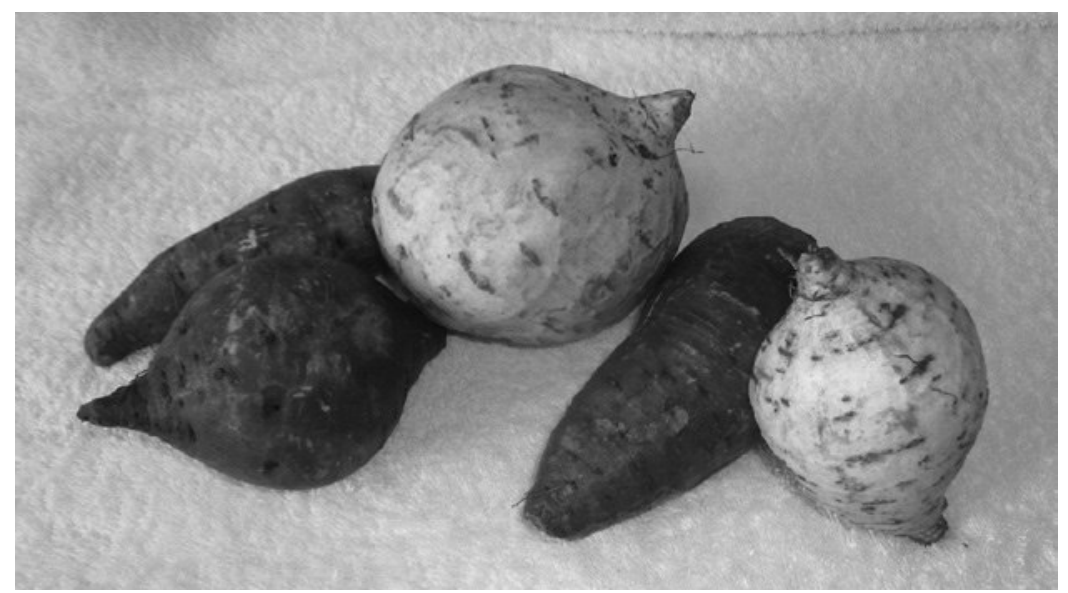

\section{RESUMEN}

Se estudió el efecto del tiempo y temperatura de escaldado sobre la actividad enzimática de la polifenoloxidasa en dos variedades de batata (blanca y colorada), con el objetivo de obtener y evaluar el comportamiento de esta enzima, las muestras fueron sometidas a temperaturas de escaldado $\left(75,80\right.$ y $\left.85^{\circ} \mathrm{C}\right)$ y tiempos de 30,60 , 90, 120, 150 y 180 s, en agua destilada con contenido de 0,5\% ácido cítrico y 1\% ácido ascórbico, regulando pH y control de oxidación. En la evaluación del efecto-temperatura (extracto) la actividad de la enzima polifenoloxidasa inicialmente en variedad colorada era de 0,263 $\Delta \mathrm{UAbs} \mathrm{min}^{-1} \mathrm{mg}^{-1}$ de proteína (variación de unidades de absorbancia por minuto por gramos de proteína), la cual disminuyó a valores de $0,0347 \Delta \mathrm{UAbs}_{\mathrm{min}}{ }^{-1} \mathrm{mg}^{-1}$ de proteína a la temperatura de $85^{\circ} \mathrm{C}$ y $180 \mathrm{~s}$. Mientras la variedad blanca $0,128 \Delta \mathrm{UAbs} \mathrm{min}^{-1} \mathrm{mg}^{-1}$ de proteína que disminuyó a valores de $0,0177 \Delta \mathrm{UAbs} \mathrm{min}^{-1} \mathrm{mg}^{-1}$ de proteína a la misma temperatura y tiempo. Se concluye que la actividad de la enzima polifenoloxidasa se redujo en un $86,8 \%$ para la variedad colorada y $86,17 \%$ en la variedad blanca, mediante la acción del escaldado.

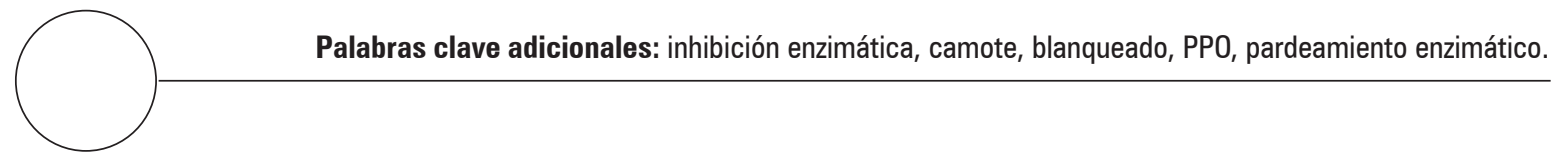

\footnotetext{
Facultad de Ingenierías, Programa de Ingeniería de Alimentos, Grupo de Investigación Procesos y Agroindustria de Vegetales, Universidad de Córdoba, Córdoba (Colombia).

2 Facultad de Ingeniería, Programa de Ingeniería Agroindustrial, Universidad de Sucre, Sincelejo (Colombia).

3 Autor para correspondencia. guillermo.arrazola@ua.es
} 


\section{ABSTRACT}

The effect of scalding time and temperature on the enzyme activity of polyphenol oxidase in two varieties of sweet potatoes (white and red) was studied with the aim of obtaining and evaluating the behavior of this enzyme. The samples were subjected to scalding temperatures $\left(75,80\right.$ and $\left.85^{\circ} \mathrm{C}\right)$ and times of 30,60 , $90,120,150,180 \mathrm{~s}$ in distilled water containing $0.5 \%$ citric acid and $1 \%$ ascorbic acid, regulating the $\mathrm{pH}$ and controlling the oxidation. In the evaluation of the temperature effect, the activity of the enzyme polyphenol oxidase in the red variety was initially $\Delta U$ UAbs $0.263 \mathrm{~min}^{-1} \mathrm{mg}^{-1}$ protein (variation of absorbance units per minute per gram of protein), which decreased to $0.0347 \Delta \mathrm{UAbs} \mathrm{min}^{-1} \mathrm{mg}^{-1}$ protein at the temperature of $85^{\circ} \mathrm{C}$ and $180 \mathrm{~s}$. The white variety had a value of $\Delta$ UAbs $0.128 \mathrm{~min}^{-1} \mathrm{mg}^{-1}$ protein, which decreased to values of $\Delta$ UAbs $0.0177 \mathrm{~min}^{-1} \mathrm{mg}^{-1}$ protein at the same temperature and time. It was concluded that the activity of the enzyme polyphenol oxidase decreased by $86.8 \%$ in the red variety and $86.2 \%$ in the white variety through the blanching action.

Additional keywords: enzyme inhibition, camote, bleached, PPO, enzymatic browning.

Fecha de recepción: 23-01-2016

Aprobado para publicación: 14-05-2016

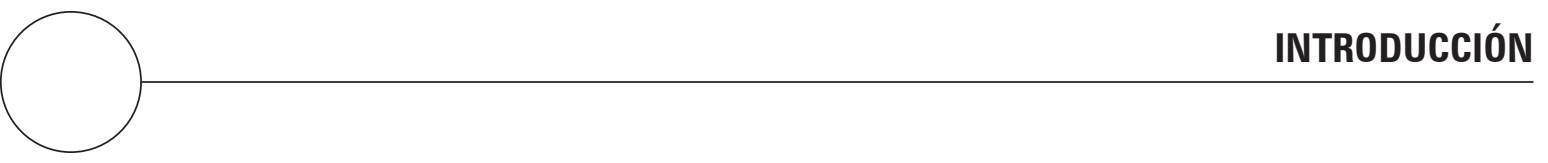

La batata (Ipomoea batatas Lam.), conocida también como camote, se ubica entre los tres principales tubérculos a nivel mundial, sosteniendo la quinta ubicación entre los alimentos que aportan mayor cantidad de energía al ser humano, después del arroz, el trigo, el maíz y la yuca. La batata se cultiva en más de 100 países en desarrollo, figurando entre los cinco cultivos más importantes en más de 50 de ellos (Arrieta y Gutiérrez, 1995; IPC, 2010). La batata produce raíces tuberosas que se emplean para el consumo directo y para procesos industriales en la obtención de almidón, harinas, postres, bebidas alcohólicas, alimentos procesados y etanol como biocombustible (Hernández et al., 2008). En la industria de alimentos, las harinas deshidratadas pueden emplearse como ingredientes funcionales, empleando la harina como materia prima cruda para procesar ponqués, biscochos, galletas, productos extruidos, chips fritos, helados, alimentos para el desayuno, papillas y alimentos para bebé (Truong y Avula, 2010), postres y helados (Martí et al., 2011), como espesante de bebidas lácteas (Andrade y Martins, 2002). Las batatas coloradas son una fuente potencial de colorantes naturales que pueden ser empleadas como fuente de aditivos en alimentos (Cuevas et al., 2011).

En el procesamiento de vegetales los cambios de color por pardeamiento enzimático, caramelización, reacción de Maillard y la oxidación de nutrientes suelen generar pigmentos provocando un aspecto desagradable frente al consumidor (Hernández, 2009) y posteriores pérdidas económicas, por el rechazo de los productos por los posibles consumidores. Entre las enzimas que catalizan estas reacciones está el polifenoloxidasa (PPO) (Casado et al., 2005; Guerrero, 2009). El polifenoloxidasa (E.C. 1.14.18.1) es una 
metaloenzima que contiene dos átomos de cobre en el sitio activo que catalizan dos tipos de reacciones usando $\mathrm{O}_{2}$ como agente oxidante: (a) la o-hidroxilación de monofenoles para producir o-difenoles y (b) la posterior oxidación de odifenoles a o-quinonas (Guillou, 2012). Entre los tratamientos para inactivar esta enzima están el uso de aditivos químicos, antioxidantes como vitaminas, agentes reductores como la cisteína, remoción de algún catalizador, irradiación y el escaldado que consiste en un tratamiento térmico medio aplicado a los vegetables por contacto con vapor o agua caliente en diferentes periodos. Se han llevado a cabo estudios para obtener las condiciones para inactivar la PPO en manzanas (Rocha y Morais 2001), pitaya amarilla (Castro et al., 2006), clavel (Mayorga e Higuera, 2007), aguacate (Amaya et al., 2008), gel de sábila (Cob et al., 2010), pera (Gasull y Becerra, 2010), papa amarilla (Trujillo et al., 2011), guacamole (Orozco et al., 2012), naranjilla (Samaniego et al., 2014, Montenegro, 2015), chicozapote (Vargas et al., 2015), entre otras.

El objetivo del presente trabajo fue evaluar el efecto del escaldado sobre la actividad enzimática de la polifenoloxidasa en dos variedades de batata (Ipomoea batata Lam.) blanca y colorada.

\section{MATERIALES Y MÉTODOS}

\section{Obtención de las muestras de batata}

Las batatas (Ipomoea batatas Lam.) de las variedades blanca y colorada se seleccionaron de las provenientes del Banco de Germoplasma de la Universidad de Córdoba. Se recolectaron $30 \mathrm{~kg}$ de muestra con tamaños y pesos semejantes. Todas las unidades de cada muestra fueron lavadas, peladas y cortadas con un potato cutter en forma de paralelepípedo de $2 \times 2 \times 4 \mathrm{~cm}$ de longitud, con el fin de estar adecuadas para la aplicación del escaldado (Alvis et al., 2008). Se utilizó agua des- tilada con contenido de $0,5 \%$ de ácido cítrico y $1 \%$ de ácido ascórbico, con el fin de reducir el pardeamiento enzimático previo al tratamiento térmico.

\section{Tratamiento de escaldado con agua caliente de las muestras de batatas}

El proceso de escaldado por inmersión en agua caliente se llevó a cabo en una freidora de acero inoxidable de $5 \mathrm{~L}$. Se sumergieron simultáneamente seis trozos de batata para cada tiempo de escaldado establecido, el producto se extrajo del baño y se secó para eliminar el agua superficial. Este procedimiento se realizó por triplicado. Las temperaturas de escaldado fueron 75,80 y $85^{\circ} \mathrm{C}$ y tiempos de proceso de 30, 60, 90, 120, 150, 180 s. Luego de realizado el tratamiento, las muestras se conservaron a $4^{\circ} \mathrm{C}$, para ser utilizadas en los procedimientos requeridos. Se dejó una muestra testigo sin procesar.

\section{Preparación del extracto enzimático}

La extracción de la enzima polifenoloxidasa en la batata se llevó a cabo siguiendo el procedimiento de Gauillard y Richard-Forget (1997). La enzima fue extraída a partir de las muestras escaldadas a las diferentes temperaturas y tiempos especificados anteriormente, en una relación $1 \mathrm{~g}$ de muestra con $10 \mathrm{~mL}$ buffer fosfato $0,2 \mathrm{M}$ con pH 6,5. La mezcla se agitó a $4^{\circ} \mathrm{C}$ durante 12 h en un agitador eléctrico. La suspensión se centrifugó a $4.000 \mathrm{rpm}$ durante $15 \mathrm{~min}$. Luego de la separación del sólido, el sobrenadante colectado se centrifugó nuevamente a $14.000 \mathrm{rpm}$ por $15 \mathrm{~min}$ en una centrífuga refrigerada (Hettich Zentrifugen, Tuttlingen, Alemania) ubicada en el Instituto de Investigaciones Biológicas del Trópico de la Universidad de Córdoba (Montería, Colombia), posteriormente fue recolectado el extracto de la enzima utilizado para realizar los ensayos, se realizó posteriormente el almacenamiento del extracto a $4^{\circ} \mathrm{C}$. 


\section{Determinación de la actividad de polifenoloxidasa}

Las unidades de actividad enzimática de polifenoloxidasa se determinaron espectrofotométricamente a $420 \mathrm{~nm}$ y $30^{\circ} \mathrm{C}$ utilizando el espectrofotómetro Genesys 20, aplicando el método de Pizzocaro et al. (1993). En una celda se colocaron 0,5 $\mathrm{mL}$ de extracto enzimático, $1 \mathrm{~mL}$ de solución de catecol al $1 \%$ y $2 \mathrm{~mL}$ de buffer fosfaton 0,2 $\mathrm{M}$, que actúa como regulador del $\mathrm{pH}$ a neutro. Se usó una celda de referencia con soluciones iguales a la anterior pero que contenía $0,5 \mathrm{~mL}$ de agua en lugar de extracto enzimático. Se midió la absorbancia durante $3 \mathrm{~min}$, en intervalos de 1 min. La actividad enzimática se expresó como $\Delta$ UAbs min $^{-1} \mathrm{mg}^{-1}$ proteína (variación de unidades de absorbancia por minuto por gramo de proteína).

El porcentaje de inhibición (\%Inh) de PPO se determinó mediante la siguiente ecuación:

$$
\% \operatorname{Inh}=\frac{\left(A_{0}-A\right)}{A_{0}} \times 100
$$

Donde la actividad de la PPO es: $A_{0}$ del extracto crudo y A en cada condición temperatura-tiempo.

\section{Inactivación térmica}

La inactivación de la PPO se determinó acorde a Manohan y Chen Wai (2012), determinando la velocidad específica (k) de inactivación térmica por la pendiente del modelo de primer orden acorde a la siguiente ecuación:

$$
\log \left(\frac{A}{A_{0}}\right)=(k / 2.303) t
$$

Donde $\mathrm{A}_{0}$ es la actividad inicial y $\mathrm{A}$ es la actividad después del calentamiento en el tiempo $t$.
El tiempo medio de la PPO se calculó siguiendo la ecuación:

$$
t_{1 / 2}=0,693 / k
$$

El tiempo de reducción decimal (D) fue estimado con la relación entre k y D acorde a la ecuación:

$$
D=(\operatorname{Ln}(10)) / k
$$

\section{Diseño experimental}

Para el análisis estadístico de las muestras, se utilizó análisis de varianza (ANOVA) con un nivel de significancia de $5 \%$, a través del software SAS 9,1 prueba de rangos múltiples (Tukey, $P \leq 0,05$ )

\section{RESULTADOS Y DISCUSIÓN}

El extracto crudo de la batata colorada presentó una actividad enzimática de la PPO de 0,263 $\Delta$ UAbs min $^{-1} \mathrm{mg}^{-1}$ proteína y la variedad blanca

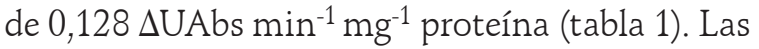
variaciones de la actividad enzimática son afectadas por las condiciones de extracción, estado fisiológico de la fruta, grado de madurez, presencia de catalizadores, concentración de la enzima (Mejía et al., 2014; Mogollón et al., 2010). Gao et al. $(2014 a, b)$ reportaron diferencias en la actividad polifenoloxidasa en batata púrpura en función de la parte estructural y de la concentración de la enzima; en este caso la presencia de una mayor cantidad de pigmentos beta-carotenoides, compuestos fenólicos y flavonoides pudo favorecer la mayor actividad en la batata colorada, mientras Hee Kim et al. (2015) reportaron mayor actividad PPO en raíces fibrosas y gruesas de batata. Compuestos fenólicos de batata incluyen ácidos clorogénicos, el color y variedad pueden influir en los niveles y perfiles de este compuesto, así una variedad de pulpa blanca que contiene 
el más alto nivel de ácido clorogénico, mientras que una variedad de color púrpura tiene niveles más bajos (Cevallos y Cisneros, 2003).

En este estudio, al comparar la actividad enzimática entre las dos variedades estudiadas (colorada y blanca) se observan diferencias significativas $(P \leq 0,05)$ y disminución de la actividad enzimática a las condiciones de temperatura-tiempo en el tratamiento de escaldado (tabla 1).

La actividad enzimática de la polifenoloxidasa en la batata variedad colorada sometida a temperaturas de 75,80 y $85^{\circ} \mathrm{C}$ y tiempos de 30 a $180 \mathrm{~s}$ en el escaldado, se puede observar que no existen diferencias significativas en los tratamientos a 80 y $85^{\circ} \mathrm{C}$ a los tiempos de 120 y 150 s, mientras para $75^{\circ} \mathrm{C}$ a los mismos tiempos sí se presentan diferencias. En la variedad Blanca muestra que no existe diferencia significativa entre 90 y $120 \mathrm{~s}$, a una temperatura de $80^{\circ} \mathrm{C}$. Estudios similares en melocotón (Brandelli y López, 2005), aguacate (Amayaet al., 2008) ybatata (Ciprianoet al., 2015).

La inhibición máxima de la actividad PPO se logra cuando el extracto es sometido a condiciones de escaldado de $85^{\circ} \mathrm{C}$ y $180 \mathrm{~s}$ con una disminución del $86,8 \%$ en la batata variedad colorada y del $86,17 \%$ en la variedad blanca (tabla 2). Liu et al. (2015) reportaron una degradación del $90 \%$ de la $\mathrm{PPO}$ en batata púrpura cuando la escaldaron con agua caliente $\left(98^{\circ} \mathrm{C}\right)$, vapor y microondas
(700 W) por un tiempo de 130, 110 y 60 s respectivamente. La PPO mostró mayor sensibilidad a los cambios de temperatura, resultado similar reportaron Gao et al. (2014b, c) en su estudio de la estabilidad térmica de la PPO en la batata colorada, diferentes de los resultados obtenidos en este estudio. Gasull y Bacerra (2010) encontraron que la $\mathrm{PPO}$ presente en manzana disminuye un $40 \%$ con un tratamiento a $50^{\circ} \mathrm{C}$ durante 20 min y en la pera a $70^{\circ} \mathrm{C}$ por $30 \mathrm{~min}$. Resultados que se encuentran alejados de los obtenidos en esta investigación.

El perfil del tratamiento de escaldado con la actividad enzimática de la PPO en la batata de variedad colorada y blanca (figura 1) muestra un comportamiento decreciente de la actividad en función del tiempo y temperatura de escaldado. Los tratamientos térmicos con temperaturas que alcanzan hasta $105^{\circ} \mathrm{C}$ se ha usado para inactivar enzimas oxidasas, teniendo en cuenta la actividad óptima de las enzimas polifenoloxidasa la cual es aproximadamente de $40^{\circ} \mathrm{C}$ (Yoruk y Marshall, 2003). Los parámetros de inactivación térmica de la PPO a un tiempo de 180 s de escaldado para las temperaturas de 75,80 y $85^{\circ} \mathrm{C}$ (tabla 3), la velocidad específica de inactivación (k) aumenta con el incremento de la temperatura, indicando que la $\mathrm{PPO}$ es menos termoestable con el incremento de la temperatura independiente de la variedad. El tiempo de vida medio $\left(t_{1 / 2}\right)$ corresponde al tiempo para reducir la actividad de

Tabla 1. Efecto del escaldado en la actividad de la polifenoloxidasa en dos variedades de batata.

\begin{tabular}{|c|c|c|c|c|c|c|}
\hline \multirow{3}{*}{ Tiempo (s) } & \multicolumn{6}{|c|}{ Actividad enzimática ( $\triangle$ UAbs $\mathrm{min}^{-1} \mathrm{mg}^{-1}$ de proteína) } \\
\hline & \multicolumn{2}{|c|}{$75^{\circ} \mathrm{C}$} & \multicolumn{2}{|c|}{$80^{\circ} \mathrm{C}$} & \multicolumn{2}{|c|}{$85^{\circ} \mathrm{C}$} \\
\hline & Colorada & Blanca & Colorada & Blanca & Colorada & Blanca \\
\hline 30 & 0,1243 a & 0,0537 a & 0,0863 a & $0,0460 \mathrm{a}$ & 0,0657 a & $0,0343 a$ \\
\hline 60 & 0,0917 b & $0,0510 \mathrm{~b}$ & $0,0713 b$ & $0,0423 b$ & $0,0580 \mathrm{~b}$ & $0,0300 \mathrm{~b}$ \\
\hline 90 & 0,0707 c & $0,0470 \mathrm{c}$ & $0,0610 \mathrm{c}$ & $0,0370 \mathrm{c}$ & $0,0490 \mathrm{c}$ & $0,0263 \mathrm{c}$ \\
\hline 120 & $0,0627 \mathrm{~d}$ & $0,0443 d$ & $0,0550 \mathrm{~d}$ & $0,0350 \mathrm{c}$ & $0,0420 \mathrm{~d}$ & $0,0237 d$ \\
\hline 150 & 0,0563 e & 0,0403 e & $0,0527 d$ & $0,0327 d$ & $0,0390 \mathrm{~d}$ & $0,0210 \mathrm{e}$ \\
\hline 180 & $0,0467 \mathrm{f}$ & $0,0380 \mathrm{f}$ & 0,0417 e & $0,0303 \mathrm{e}$ & 0,0347 e & $0,0177 f$ \\
\hline
\end{tabular}

Promedios con letras distintas indican diferencia significativa según la prueba de Tukey $(P \leq 0,05)$. 
la PPO a la mitad de la inicial, reduciéndose con el incremento de la temperatura y siendo superior en la batata variedad blanca, indicando una mayor estabilidad de la enzima a $75^{\circ} \mathrm{C}$ y $80^{\circ} \mathrm{C}$ donde se observan diferencias mayores del indicador con la variedad colorada.

El tiempo de reducción decimal (D) requerido para reducir en un $90 \%$ la actividad inicial de la PPO estuvo del orden superior a los $300 \mathrm{~s} \mathrm{a} 75^{\circ} \mathrm{C}$ en la variedad blanca y en todas las condiciones temperatura superior a la mostrada en la variedad colorada.

Estos valores son inferiores a los reportados por Manohan y Chen Wai (2012) en batata colorada con (D) entre 305,7 min a 35 min en el rango de 60 a $75^{\circ} \mathrm{C}$. El parámetro (Z) se relaciona inversamente con la sensibilidad al calor de la enzima; en general, el bajo valor del parámetro $(Z)$ en la variedad blanca indica una gran sensibilidad al calor respecto a la mostrada en la variedad colorada. La PPO en la variedad blanca muestra mayor sensibilidad a las temperaturas de 75 y $80^{\circ} \mathrm{C}$; sin embargo, a $85^{\circ} \mathrm{C}$ es comparativamente igual con la actividad en la variedad colorada. Estas di- ferencias en la cinética de inactivación por calor pueden estar asociadas a su diferente composición producto de las condiciones agronómicas y climáticas bajos las cuales se desarrolló la batata. La menor actividad de la PPO en ambas variedades obtenidas bajo condiciones de tiempo y temperaturas en las que se alcanza una mayor reducción, se pueden catalogar como favorables para posteriores procesos de transformación, como fritura, secado y obtención de harinas. La técnica apropiada de escaldado permite obtener productos fritos de batata de alta calidad sin un pardeamiento que reduzca la aceptabilidad del consumidor.

\section{CONCLUSIONES}

En la evaluación del efecto tiempo-temperatura sobre la reducción de la enzima polifenoloxidasa en la variedad colorada, inicialmente esta se encontraba en valores de $0,263 \Delta$ UAbs min $^{-1} \mathrm{mg}^{-1}$ de proteína disminuyendo a valores de 0,0347 $\Delta$ UAbs $\mathrm{min}^{-1} \mathrm{mg}^{-1}$ de proteína a la temperatura de $85^{\circ} \mathrm{C}$ a $180 \mathrm{~s}$. Mientras que para la variedad blanca 0,128 $\Delta$ UAbs $\mathrm{min}^{-1} \mathrm{mg}^{-1}$ de proteína dis-
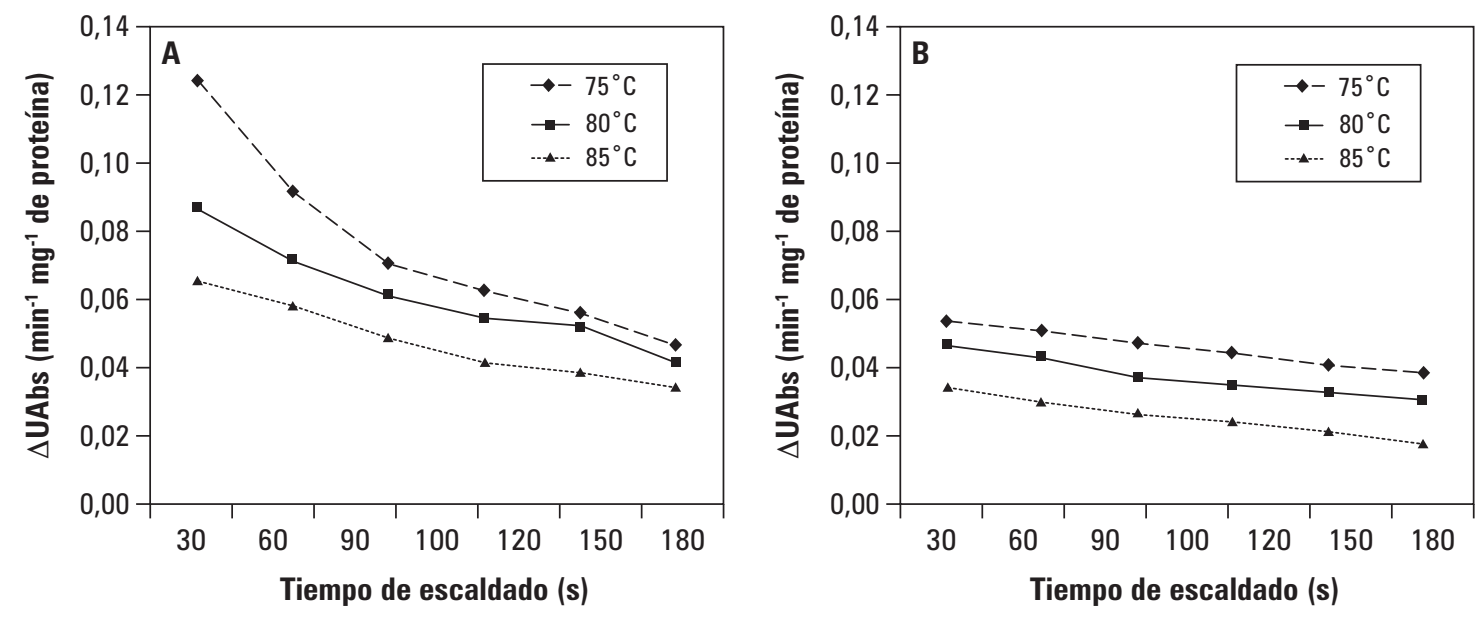

Figura 1. Actividad de la PPO en las variedades de batata Colorada (A) y Blanca (B) a $75^{\circ} \mathrm{C}, 80^{\circ} \mathrm{C}$ y $85^{\circ} \mathrm{C}$. 
Tabla 2. Inhibición de la actividad polifenoloxidasa en dos variedades de batata.

\begin{tabular}{|c|c|c|c|c|c|c|}
\hline \multirow{2}{*}{ Tiempo (s) } & \multicolumn{7}{|c|}{ Inhibición (\%) } \\
\cline { 2 - 7 } & \multicolumn{2}{|c|}{$75^{\circ} \mathrm{C}$} & \multicolumn{2}{c|}{$80^{\circ} \mathrm{C}$} & \multicolumn{2}{c|}{$85^{\circ} \mathrm{C}$} \\
\cline { 2 - 7 } & Colorada & Blanca & Colorada & Blanca & Colorada & Blanca \\
\hline 30 & 52,73 & 58,04 & 67,18 & 64,06 & 75,01 & 73,20 \\
\hline 60 & 65,13 & 60,15 & 72,88 & 66,95 & 77,94 & 76,56 \\
\hline 90 & 73,11 & 63,28 & 76,80 & 71,09 & 81,36 & 79,45 \\
\hline 120 & 76,15 & 65,39 & 79,08 & 72,65 & 84,03 & 81,48 \\
\hline 150 & 78,59 & 68,51 & 79,96 & 74,45 & 85,17 & 83,59 \\
\hline 180 & 82,24 & 70,31 & 84,14 & 76,32 & 86,80 & 86,17 \\
\hline
\end{tabular}

Tabla 3. Parámetros de la inactivación térmica en dos variedades de batata.

\begin{tabular}{|c|c|c|c|c|c|c|}
\hline \multicolumn{2}{|c|}{ Variedad } & \multicolumn{3}{c|}{ Colorada } & \multicolumn{3}{c|}{ Blanca } \\
\hline Temperatura $\left({ }^{\circ} \mathrm{C}\right)$ & $\mathrm{k}(1 / \mathrm{s})$ & $\mathrm{t}_{1 / 2}(\mathrm{~s})$ & $\mathrm{D}(\mathrm{s})$ & $\mathrm{k}(1 / \mathrm{s})$ & $\mathrm{t}_{1 / 2}(\mathrm{~s})$ & $\mathrm{D}(\mathrm{s})$ \\
\hline 75 & 0,010 & 72,16 & 239,75 & 0,007 & 102,70 & 341,22 \\
\hline 80 & 0,010 & 67,72 & 225,01 & 0,008 & 86,56 & 287,60 \\
\hline 85 & 0,011 & 61,58 & 204,60 & 0,011 & 63,04 & 209,45 \\
\hline $\mathrm{Z}\left({ }^{\circ} \mathrm{C}\right)$ & 144,93 & 47,17 & & & & \\
\hline
\end{tabular}

minuye a valores de $0,0177 \Delta$ UAbs $\mathrm{min}^{-1} \mathrm{mg}^{-1}$ de proteína a la misma temperatura y tiempo de escaldado. Al comparar la actividad enzimática de las dos variedades de estudio se evidencia la reducción de la polifenoloxidasa en un $86,8 \%$ para la variedad colorada y un $86,17 \%$ en la variedad blanca. Con estos resultados se presenta una alternativa de conservación para la batata y sus derivados en la obtención de otros productos como chips y extruidos, principalmente.

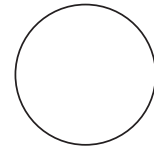

Alvis, A., C. Vélez y M. Rada-Mendoza. 2008. Composición de ñames frescos cultivados en Colombia y sometidos a freído por inmersión. Información Tecnológica 19(1), 1-10. Doi: 10.4067/ S0718-07642008000100002

Amaya, E., R. Tarkus y M. Domínguez. 2008. Extracción y caracterización cinética de la enzima polifenoloxidasa del aguacate (Persea americana Miller) var. Hass. Revista de la Facultad de Ingeniería Química 47, 10-16.

Andrade, R. y J. Martins. 2002. Influência da adição da fécula de batata-doce (Ipomoea batatas Lam) sobre

\section{REFERENCIAS BIBLIOGRÁFICAS}

a viscosidade do permeado de soro de queijo. Cienc. Tecnol. Aliment. 22(3), 249-253. Doi: 10.1590/ S0101-20612002000300009

Arrieta, W. y R. Gutiérrez R. 2009. Monografía del cultivo de la Batata (Ipomoea batatas Lam). Universidad de Córdoba, Montería, Colombia.

Brandelli, A. y C. López. 2005. Polyphenoloxidase activity, browning potential andphenolic content of peaches during postharvest ripening. J. Food Biochem. 29(16) 624-637. Doi: 10.1111/j.17454514.2005.00026.x 
Casado, J., S. Selles y R. Bru. 2005. Purificación y caracterización cinética de la polifenoloxidasa (PPO) de frutos de tomate (Lycopersicon esculentum cV. Muchamiel). J. Food Biochem. 29(4), 381-401. Doi: 10.1111/j.1745-4514.2005.00037.x

Castro, J.A., L.E. Baquero y C.E. Narváez. 2006. Catalasa, peroxidasa y polifenoloxidasa de pitaya amarilla (Acanthocereus pitajaya). Rev. Colomb. Quim. 35(1) 91-101.

Cevallos, C. y Z. Cisneros. 2003. Estudios estequiométricos y cinéticos de antioxidantes fenólicos de Andina maíz morado y el camote de carne roja. J. Agr. Food Chem. 5, 3313-3319.

Cipriano, P., L. Ekici, R. Barnes, C. Gomes y S.Talcott. 2015. Pre-heating and polyphenol oxidase inhibition impact on extraction of purple sweet potato anthocyanins. Food Chem. 180, 227-234. Doi: 10.1016/j.foodchem.2015.02.020

Cob, N., J. Tello, J. Escamilla, L. Vargas y J. Tamayo. 2010. Actividad de la polifenoloxidasa en el gel de Sábila (Aloe vera Mill). Rev. Iber. Tecnol. Postcosecha 11(2), 221-227.

Cuevas, E., S. Hillebrand y P. Winterhalter. 2011. Anthocyanins in Purple Sweet Potato (Ipomoea batatas L.) varieties. Fruit Veg. Cereal Sci. Biotechnol. 5(special issue 2), 19-24.

Gao, L., L.C. Zhao, J.D. Duan y Y.L. Tao. 2014a. Study on the process of the reaction catalyzed by polyphenol oxidase from purple sweet potato. Adv. Mat. Res. 898, 153-156. Doi: 10.4028/www.scientific.net/AMR.898.153

Gao, L., Y. Yuan, Y.C. Li y Y.X. Wei. 2014b. Study on polyphenol oxidase activity in different parts of purple sweet potato. Adv. Mat. Res. 898, 149-152. Doi: 10.4028/www.scientific.net/AMR.898.149

Gao, L., L. Lin, L.C. Zhao y F. Wu. 2014c. Study on heat stability of polyphenol oxidase from purple sweet potato. Appl. Mechanics Mat. 716-717, 122 125. Doi: 10.4028/www.scientific.net/AMM.716717.122

Gasull, E. y D. Becerra. 2010. Caracterización de polifenoloxidasa extraída de pera (cv. Packam's Triumph) y manzana (cv. Red Delicious). Información Tecnológica 17(6), 69-74.

Gauillard, F. y F. Richard-Forget. 1997. Polyphenoloxidase from Williams pear (Pyrus communis L., cv. Williams): activation, purification and some properties. J. Food Sci. 74, 49-56. Doi: 10.1002/(SICI)1097-0010(199705)74:1<49::AIDJSFA769>3.0.CO;2-K
Guerrero, C. 2009. Inhibición de la actividad enzimática de la polifenoloxidasa extraída del banano (Cavendish valery) mediante sistemas bifásicos acuosos con isoespintanol y ácido ascórbico. Tesis de doctorado. Universidad Nacional de Colombia, Medellín, Colombia.

Guillou, N. 2012. Mecanismos y efectos asociados a procesos de oxidación de compuestos fenólicos en vinos. Trabajo de grado. Facultad de Ciencias Agronómicas, Universidad de Chile, Santiago.

Hernández, C. 2009. Acción y efectos de la polifenoloxidasa en alimentos. Universidad Veracruzana, Xalapa Enríquez, México.

Hernández, M., J. Torruco, L. Chel y D. Betancur. 2008. Caracterización fisicoquímica de almidones de tubérculos cultivados en Yucatán, México. Ciênc. Tecnol. Aliment. 28(3), 718-726. Doi: 10.1590/ S0101-20612008000300031

IPC (International Potato Center). 2010. La batata en cifras: producción, utilización, consumo, alimentación. En: http://www.cipotato.org/sweetpotato/links.asp y http://.cipotato.org./sweetpotato/ facts/batacif.pdf; consulta: abril de 2015.

Kim, Y.-H., S.-C. Park, C.Y. Ji, J.J. Lee, J.C. Jeong, H.-S. Lee y S.-S. Kwak. 2015. Diverse antioxidant enzyme levels in different sweet potato root types during storage root formation. Plant Growth Regul. 75(1), 155-164. Doi: 10.1007/s10725-014-9940-x

Liu, P., A. Mujumdar, M. Zhang y H. Jiang. 2015. Comparison of three blanching treatments on the color and anthocyanin level of the microwave-assisted sprouted bed drying of purple flesh sweet potato. Drying Technol. 33, 66-71. Doi: 10.1080/07373937.2014.936558

Manohan, D. y W. Chen Wai. 2012. Characterization of polyphenol oxidase in sweet potato (Ipomoea batatas L.). J. Advancement Sci. Arts 3(1), 14-31.

Martí, H.R., H. D’Chudil y G. Corbino. 2011. La batata: El redescubrimiento de un cultivo. Ciencia Hoy 21(121), 17-23.

Mayorga, V. y B. Higuera. 2007. Aislamiento y caracterización de una polifenoloxidasa relacionada con la tolerancia del clavel (Dianthus caryophyllus) a Fusarium oxysporum sp. dianthi raza 2. Acta Biol. Colomb. 12(2), 81-94.

Mejía, C., D. Gaviria, R. Bru, L. Rengifo, Á. Alegría y E. Aguilar. 2014. Caracterización cinética de la enzima polifenoloxidasa en seis estadios de maduración en lulo (Solanum quitoense Lam.) var. Castilla. Actu. Biol. 36 (101), 107-117. 
Mogollón, P., P. Lizarazo y Y. Quintero. 2010. Determinación de la actividad e inhibición de la polifenoloxidasa en el lulo (Solanum quitoense Lam.). @ Limentech Cienc. Tecnol. Aliment. 8(2), 112-117.

Montenegro, L. 2015. Estudio de la Inhibición del pardeamiento enzimático por irradiación y de la calidad poscosecha durante el almacenamiento refrigerado de la naranjilla (Solanum quitoense Lam.) irradiada. Trabajo de grado. Facultad de Ingeniería Quimica y Agroindustrial, Escuela Politécnica Nacional, Quito.

Orozco, M., R. Prieto y L. Díaz. 2012. Efecto de la adición de cebolla, ajo y la aplicación de ultrasonido de alta intensidad sobre la actividad de la polifenoloxidasa en guacamole. Vitae 19(Supl. 1), S132-S134.

Pizzocaro, F., D. Torreggiani y G. Gilardi. 1993. Inhibition of apple polyphenoloxidase (PPO) by ascorbic acid, citric acid and sodium chloride. J. Food Proc. Pres. 17, 21-30. Doi: 10.1111/j.1745-4549.1993.tb00223.x

Rocha, A. y A. Morais. 2001. Characterization of polyphenoloxidase (PPO) extracted from 'Jonagored' apple. Food Control 12, 85-90. Doi: 10.1016/ S0956-7135(00)00026-8

Samaniego, E., A. Ibarz y J. Ruales. 2014. Efecto de la irradiación ultravioleta en la actividad enzimática de la polifenoloxidasa y peroxidasa y las propiedades fisicoquímicas del jugo de dos variedades de Naranjilla (Solanum quitoense Lam.). Revista EPN 33(2).

Trujillo N., O. Urrutia y J. Pabón. 2011. Evaluación preliminar del efecto del $\mathrm{pH}$ y de la temperatura en la actividad de la polifenoloxidasa en papa amarilla (Solanumphureja), @limentech Cienc. Tecnol. Aliment. 9(2), 161-166

Truong, V. y R. Avula. 2010. Sweet potato purees and dehydrated powders for functional food ingredients. En: http://naldc.nal.usda.gov/naldc/download.xhtml id=44182\&content=PDF; consulta: junio de 2015.

Vargas, M., E. Sauri, E. Tamayo, S. González y J. Tamayo. 2015. Fresh-cut sapodilla (Achras sapota) with antioxidant activity; a modern demand for consumption fruits, Rev. Mex. Agronegocios 36, 1315-1324.

Yoruk, R y M.R. Marshall. 2003. Physicochemical properties and function of plant polyphenol oxidase: A review. J. Food Biochem. 27, 361-422. Doi: 10.1111/j.1745-4514.2003.tb00289.x 